\title{
Pediatric Lung Transplantation
}

\author{
Anand Patel and Albert Faro
}

\section{Contents}

History

Indications

Contraindications

Disease Specific Indications and Contraindications.................................................. 763

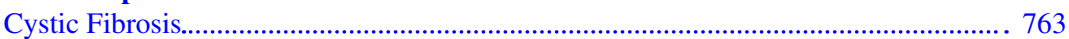

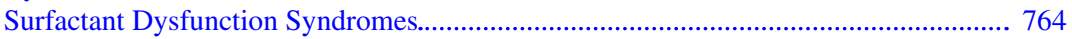

Pulmonary Vascular Disorders........................................................................................ 764

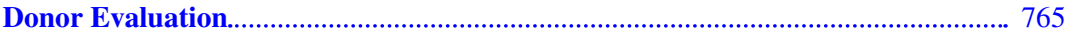

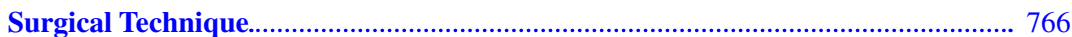

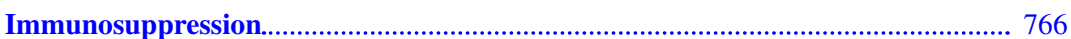

Post-operative Monitoring and Management........................................................ 767

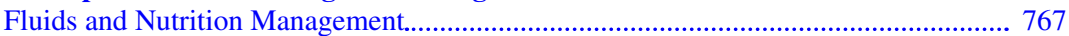

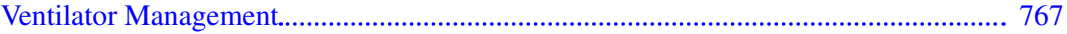

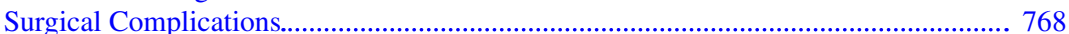

Primary Graft Dysfunction..................................................................................... 768

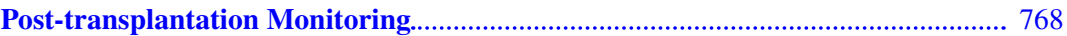

Post-transplantation Complications.................................................................... 768

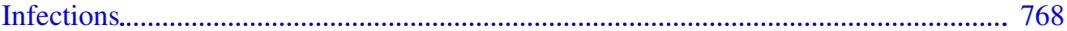

Post-transplant Lymphoproliferative Disease (PTLD) ................................................... 770

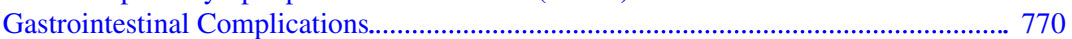

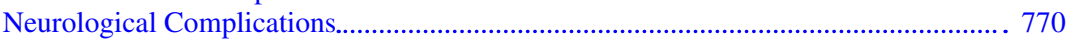

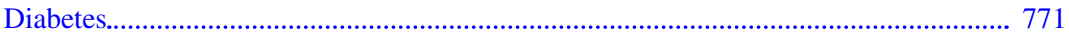

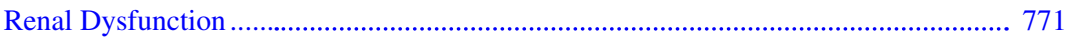

Rejection

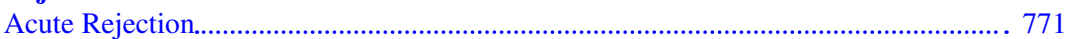

Antibody Mediated Rejection...................................................................................... 771

\footnotetext{
A. Patel $\cdot$ A. Faro $(\square)$

Washington University in St. Louis School of Medicine, St. Louis, MO, USA

e-mail: afaro@cff.org
} 


\section{History}

Dr. James Hardy performed the first lung transplant in 1963 at the University of Mississippi Medical Center in a 58 year old convict serving a life sentence. The patient succumbed to renal failure 18 days after the procedure. There were many subsequent attempts, but it was not until 1983 at the University of Toronto that Dr. Joel Cooper performed the first successful long-term lung transplant. Dr. Denton Cooley attempted the first heart-lung transplantation in a child in 1968. The patient was a 2 month old with pulmonary hypertension and a complete AV canal, but they only survived for 14 hours. The first successful isolated lung transplant in a child was performed at the University of Toronto in 1987 in a 16 year old boy with pulmonary fibrosis. The 1990s witnessed a rapid growth in the number of pediatric lung transplants performed annually. However, unlike lung transplantation in adults, that growth has not continued in the 2000s. At present, there are approximately 100 lung transplants done worldwide per year in children.

\section{Indications}

In general, lung transplantation should be considered in children suffering from life threatening end-stage lung disease or pulmonary vascular disease refractory to medical therapy. The indications in childhood vary by age. Pulmonary hypertension, secondary to congenital heart disease or other pulmonary vascular disorders, is the leading indication in infancy followed by interstitial lung disease, including surfactant protein abnormalities. For older children and adolescents cystic fibrosis (CF) accounts for half to three quarters of all lung transplant procedures. Although the most common diagnoses for children receiving lung transplants are listed in Table 74.1 determining which children may benefit from lung transplantation is often not as straightforward. In addition, determining the appropriate time to proceed is often challenging. Transplant physicians thoughtfully gage when a patient has entered the transplant window. This is the point in time where the patient is sick enough to warrant lung transplantation but not so sick that the odds of a good outcome have markedly deteriorated. Multiple variables must be carefully considered including the trajectory of the underlying disease. Lung transplantation is a procedure of last resort and, when performed, should provide the patient with not

Table 74.1 Common indications for pediatric lung transplantation by age group

\begin{tabular}{|l|r|}
\hline Infants (<1 year of age) & \\
\hline Congenital heart disease & $16.7 \%$ \\
\hline Surfactant protein B deficiency & $16.7 \%$ \\
\hline Idiopathic pulmonary hypertension & $12.5 \%$ \\
\hline Idiopathic pulmonary fibrosis & $10.4 \%$ \\
\hline Pulmonary vascular disease & $8.3 \%$ \\
\hline Other pulmonary fibrosis & $7.3 \%$ \\
\hline Preschool children (age 1-5 years) & \\
\hline Idiopathic pulmonary hypertension & $22.4 \%$ \\
\hline Idiopathic pulmonary fibrosis & $16.8 \%$ \\
\hline Other pulmonary fibrosis & $8.8 \%$ \\
\hline Retransplantation & $8.8 \%$ \\
\hline Congenital heart disease & $8.0 \%$ \\
\hline Bronchiolitis obliterans (not retransplant) & $8.0 \%$ \\
\hline Pulmonary vascular disease & $5.6 \%$ \\
\hline School aged children (age 6-10 years) & \\
\hline Cystic fibrosis & $53.0 \%$ \\
\hline Idiopathic pulmonary hypertension & $8.7 \%$ \\
\hline Bronchiolitis obliterans (not retransplant) & $6.8 \%$ \\
\hline Retransplantation & $6.4 \%$ \\
\hline Idiopathic pulmonary fibrosis & $5.7 \%$ \\
\hline Adolescents (age 11-17 years) & \\
\hline Cystic fibrosis & $70.6 \%$ \\
\hline Idiopathic pulmonary hypertension & $7.8 \%$ \\
\hline Retransplantation & $5.3 \%$ \\
\hline
\end{tabular}


only an improved likelihood of survival over their underlying disease but also an improvement in their quality of life.

Lung transplantation is a lifelong commitment to a complex and demanding regimen that includes daily immunosuppressive therapy, frequent physician visits, routine surveillance procedures, including bronchoscopy, transbronchial biopsy, radiographic examinations, and blood work. Therefore, the pediatric patient must have adequate family support and access to transplant services and medications as well as demonstrate a willingness and ability to adhere to this admittedly complex regimen.

For the referring center, the correct timing of the referral to a transplant center is at times difficult to determine. Even for CF, where many investigators have rigorously studied this question, the answer remains unclear. Therefore, it is best for the referring center to consider the possibility of lung transplantation in a child whose pulmonary disease has a well defined trajectory of continued decline and whose life expectancy is severely limited by the disease process.

\section{Contraindications}

Contraindications can be divided into two categories: absolute and relative (Table 74.2). These often vary from one transplant program and the

Table 74.2 Examples of contraindications to lung transplantation (varies by transplant center)

\begin{tabular}{|c|c|}
\hline Absolute & Relative \\
\hline Active malignancy & $\begin{array}{l}\text { Poorly controlled } \\
\text { diabetes }\end{array}$ \\
\hline Multiorgan failure & Pleurodesis \\
\hline HIV & Scoliosis \\
\hline Sepsis & $\begin{array}{l}\text { Markedly abnormal } \\
\text { body mass index }\end{array}$ \\
\hline Severe neuromuscular disease & Osteoporosis \\
\hline $\begin{array}{l}\text { Documented, refractory } \\
\text { nonadherence }\end{array}$ & $\begin{array}{l}\text { Prolonged mechanical } \\
\text { ventilation }\end{array}$ \\
\hline Hepatitis C & ECMO \\
\hline $\begin{array}{l}\text { Chronic airway infection with } \\
\text { Burkholderia cenocepacia or } \\
\text { Mycobacterium abscessus }\end{array}$ & $\begin{array}{l}\text { Chronic airway } \\
\text { infection with } \\
\text { multi-drug resistant } \\
\text { organisms }\end{array}$ \\
\hline Active tuberculosis & Renal insufficiency \\
\hline
\end{tabular}

difference is based on experience, expertise, and the center's view of the available data. In other words, an absolute contraindication at one center may be a relative contraindication at another. There is general agreement that multi-organ disorders, active malignancy, or certain types of active infection are absolute contraindications to transplant. At some centers multi-organ transplantation may be possible in select individuals. This may include heart-lung transplantation and in patients with $\mathrm{CF}$ combined liver-lung transplantation.

\section{Disease Specific Indications and Contraindications}

\section{Cystic Fibrosis}

Several different models have been developed over the years to predict survival in CF; however, none has demonstrated any greater accuracy than Kerem's criteria. This model found that a forced expiratory volume in 1 second (FEV1) of less than $30 \%$ predicted was associated with an increased risk of death within 2 years; associated factors include decreased arterial oxygen tension $\left(\mathrm{PaO}_{2}\right)$, an increase in arterial carbon dioxide tension $\left(\mathrm{PaCO}_{2}\right)$ on room air, younger age, and female gender.

Specific indications Progressive lung disease and worsening quality of life despite optimal medical therapy; FEV1 $<30 \%$ predicted.

Specific contraindications Varies by individual transplant centers, but most commonly center around microbiologic concerns with Burkholderia cenocepacia constituting one of the more common absolute contraindications. Many centers now consider infection with Mycobacterium abscessus either an absolute or relative contraindication and this can vary from center to center based on whether the patient is only culture positive and smear negative or culture positive and smear positive. The data presently available suggest that outcomes for patients infected with other nontuberculous mycobacteria are not sub- 
stantially different than for patients not infected with those organisms. Other potential relative contraindications may include malnutrition, poorly controlled diabetes, osteoporosis, previous pleurodesis (especially talc), and liver failure, if the center cannot perform a combined liver-lung transplant.

\section{Surfactant Dysfunction Syndromes}

Four distinct genetic surfactant deficiency syndromes have been identified and vary in their presentation. The diagnosis can be confirmed by genetic analysis. Lung biopsy may demonstrate diffuse alveolar type II cell hyperplasia, alveolar proteinosis, and septal thickening. Surfactant protein B deficiency is an autosomal recessive disorder. It presents in the newborn period with respiratory failure and is typically lethal within the first year of life. There is no effective medical therapy. Lung transplantation is the only intervention with the potential to sustain life.

Surfactant protein $\mathrm{C}$ deficiency has a more variable presentation and is inherited in autosomal dominant pattern. The age of disease onset spans from the newborn period to adulthood. In addition, even infants presenting with more severe findings such as respiratory failure can improve over time. This variability suggests that other genetic or environmental modifiers may influence the course. Corticosteroids, hydroxychloroquine, and azithromycin have been used in isolated cases, but the results are difficult to interpret.

Adenosine triphosphate binding cassette protein member A3 (ABCA3) deficiency has two distinct ways of presenting. It can be found in newborns with respiratory failure, but a milder form also exists that is found in older children presenting with interstitial lung disease. Dense lamellar bodies are seen by electron microscopy of lung tissue.

Mutations in the NKX2.1 gene, also known as the thyroid transcription factor gene, may cause findings similar to any of the previously mentioned surfactant abnormalities as NKX2.1 is important for the expression of SP-B, SP-C, and ABCA3. Mutations in the gene can lead to brain-thyroid-lung syndrome so that patients may present with hypothyroidism or benign chorea in addition to ILD.

Specific indications Once a decision to pursue transplant has been made, patients with SPB deficiency should be transferred as soon as a possible to the transplant center. For the other surfactant processing abnormalities, lung transplantation is indicated for refractory respiratory failure or progressive respiratory insufficiency unresponsive to medical interventions.

Specific contraindications Significant neurologic injury and, at some centers, VA-ECMO.

\section{Pulmonary Vascular Disorders}

In this broad group of disorders characterized by pulmonary hypertension, we will include idiopathic pulmonary hypertension (IPH), pulmonary vein anomalies, congenital heart disease (CHD), and patients with an inadequate pulmonary vascular bed. These patients may die from progressive right heart failure, arrhythmias, or massive hemoptysis. In general, a decreased cardiac index, elevated pulmonary vascular resistance, right atrial pressures $>7.4 \mathrm{~mm} \mathrm{Hg}$, and right ventricular end diastolic pressure of $>10.4 \mathrm{~mm} \mathrm{Hg}$ predict mortality and are indications for transplant. Other factors that may impact survival include elevated von Willebrand factor, uric acid levels, and brain natriuretic peptide concentrations.

Advances in pharmacotherapy have dramatically changed the landscape for patients with IPH so that lung transplantation should only be considered in patients who have failed medical therapy. The same cannot be said for pulmonary venous anomalies, such as pulmonary vein stenosis (PVS) or pulmonary veno-occlusive disease (PVOD), which are not only poorly responsive to medical therapy but also poorly responsive to cardiac catheterization or surgical interventions.

The timing of transplantation in patients with pulmonary hypertension associated with CHD, 
including those with Eisenmenger syndrome, remains unclear. Many of these patients can live for years after diagnosis. An added decision that must be made for this group of patients is whether or not the cardiac defect can be repaired or if the patient will require a combined heart-lung transplant. Unfortunately, the need for two organs can markedly prolong the time on the waiting list. If the patient has an atrial septal defect, ventricular septal defect or patent ductus arteriosus, these are relatively simple repairs and can be performed at the time of lung transplantation.

For patients with an inadequate pulmonary vascular bed, such as those with pulmonary atresia, congenital diaphragmatic hernia, and ventricular septal defect with multiple aortopulmonary collaterals, lung transplantation is high risk, but it may be the sole life prolonging intervention.

Specific indications Idiopathic pulmonary hypertension - patients who have failed medical management

Pulmonary venous anomalies - upon making the diagnosis urgent referral is recommended

Congenital heart disease or inadequate pulmonary vascular bed - progressive severe hypoxia, syncope, massive hemoptysis

Specific contraindications Multiple prior thoracotomies associated with development of extensive collateral circulation and/or significant presence of preformed antibodies.

\section{Donor Evaluation}

Unfortunately, not only does there continue to be a shortage of donors but data from the OPTN/ SRTR database in the United States shows that, in 2012, of the 8143 organ donors only 1708 donated lungs. In other words, only $21 \%$ of organ donors had lungs that were deemed suitable for procurement. The reasons are varied and include the mechanism of death, consequences of mechanical ventilation, and donor management, but they highlight the importance of understanding what characteristics make a donor acceptable.
Beyond the basics of matching for size and blood type, the characteristics outlined in Table 74.3 may contribute to both short- and long-term outcomes and determine whether the donor lungs will be accepted by a transplant center.

Table 74.3 Ideal donor characteristics

\begin{tabular}{l|l}
$\begin{array}{l}\text { 1. Donor age } \\
\text { Ideal } \\
\text { donor }\end{array}$ & Less than 55 years old \\
\hline $\begin{array}{l}\text { Evidence } \\
\text { There is data to support poorer outcomes } \\
\text { with older donors, especially if combined } \\
\text { with ischemic times of greater than } 6 \text { hours. }\end{array}$ \\
\hline $\begin{array}{l}\text { 2. } \\
\text { Donor }\end{array}$
\end{tabular}

2. Donor ABG

Ideal $\mathrm{PaO}_{2}$ greater than 300 on $\mathrm{FiO}_{2}$ 1.0, PEEP of donor $\quad 5 \mathrm{~cm} \mathrm{H}_{2} \mathrm{O}$

Evidence Inadequate data to either support or refute this cutoff. Studies mainly focus on interventions to increase donor $\mathrm{PaO}_{2}$ to over 300 and then procure them.

3. Radiographic findings

Ideal Clear CXR

donor

Evidence No data in regard to use of abnormal chest films. If finding is suggestive of atelectasis, aggressive airway clearance and use of the bronchoscope to remove mucus plugs may salvage an otherwise unusable lung.

4. Ischemic time

Ideal 4-6 hours

donor

Evidence Reports describe successful outcomes with ischemic times from 6 to 11 hours. Poor outcomes clearly associated with older donors and prolonged ischemic times.

5. Size matching

Ideal Most centers use height and or predicted

donor TLC. Accepted ranges are plus or minus $20 \%$.

Evidence Data demonstrates no adverse effect on outcome when using donor lungs within $75-125 \%$ of recipient TLC.

\section{Airway secretions}

Ideal No purulent secretions on bronchoscopy, donor negative gram stain

Evidence Infection is a major source of early post-transplant morbidity and mortality. However, evidence suggests that a positive sputum gram stain does not correlate with the development of pneumonia. There is data to suggest that it is the amount of purulent secretions that may be of importance in predicting outcome post-transplant.

(continued) 
Table 74.3 (continued)

\begin{tabular}{|c|c|}
\hline \multicolumn{2}{|c|}{ 7. Smoking } \\
\hline $\begin{array}{l}\text { Ideal } \\
\text { donor }\end{array}$ & Less than 20 pack year history \\
\hline Evidence & $\begin{array}{l}\text { There is no data that either supports or } \\
\text { refutes this criterion. Concerns include the } \\
\text { development of malignancy post-transplant } \\
\text { as well as the potential for increased risk for } \\
\text { a poor peri-operative outcome. }\end{array}$ \\
\hline \multicolumn{2}{|c|}{ 8. Malignancy } \\
\hline $\begin{array}{l}\text { Ideal } \\
\text { donor }\end{array}$ & No cancer history \\
\hline Evidence & $\begin{array}{l}\text { Little data, particularly in donors with a past } \\
\text { history of a malignancy. The potential risk is } \\
\text { likely based on histology, tumor stage, and } \\
\text { length of cancer free survival. Primary CNS } \\
\text { tumors rarely spread. Risk factors include } \\
\text { medulloblastoma or glioblastoma, previous } \\
\text { craniotomy, venticular shunt, tumor } \\
\text { radiation. Renal cell carcinoma is the most } \\
\text { common type of cancer that is transmitted } \\
\text { from a donor. }\end{array}$ \\
\hline \multicolumn{2}{|c|}{ 9. Length of mechanical ventilation } \\
\hline $\begin{array}{l}\text { Ideal } \\
\text { donor }\end{array}$ & Ventilated for less than 3 days \\
\hline Evidence & $\begin{array}{l}\text { Greater than } 2 \text { days of mechanical } \\
\text { ventilation is a risk factor for the } \\
\text { development of ventilator associated } \\
\text { pneumonia. However, donors with } \\
\text { prolonged courses and clear X-rays and } \\
\text { good gas exchange may in fact be better } \\
\text { donors since the sequelae of aspiration may } \\
\text { not be evident within the first } 24-48 \text { hours. }\end{array}$ \\
\hline \multicolumn{2}{|c|}{ 10. Serologies } \\
\hline $\begin{array}{l}\text { Ideal } \\
\text { donor }\end{array}$ & $\begin{array}{l}\text { Seronegative for HIV and hepatitis A, B \& } \\
\text { C }\end{array}$ \\
\hline Evidence & $\begin{array}{l}\text { Higher risk of morbidity/mortality } \\
\text { associated with immunosuppression. }\end{array}$ \\
\hline
\end{tabular}

\section{Surgical Technique}

The surgical technique used in children is similar to that used in adults with the following exceptions: (i) virtually all children will require cardiopulmonary bypass, (ii) in children bilateral, as opposed to single, lung transplantation is much more common. The typical approach is a bilateral sequential procedure with a transsternal bilateral anterior thoracotomy, referred to as the clamshell incision, which provides excellent access and visualization for the procedure. At our center, an end-to-end anastomosis is favored for the airway because of the higher incidence of stenosis seen with a telescopic anastomosis. Many centers will cover the anastomosis with peribronchial lymphatic tissue. It is important to note that the bronchial circulation, lymphatic system, and nervous system are not reanastomosed. However, a few centers do advocate direct bronchial revascularization.

If a cardiac repair is necessary, it is done after the pneumonectomies but prior to implantation as this provides the surgeon with a bloodless operative field. After the second lung has been implanted, they are inflated to expand all of the atelectatic portions. The patient is then weaned off of bypass. Two chest tubes are left in each pleural space. The pulmonary veins can be assessed at this time with transesophageal echocardiography, and the pulmonary artery anastomoses can be evaluated upon arrival to cardiac intensive care unit with a perfusion scan.

Living lobar transplantation has fallen out of favor in the United States because lungs are allocated on the basis of a disease severity score rather than just time spent on the list. This has obviated the most common reason for living lobar transplantation, presenting to a transplant center in extremis and not having accrued enough time on the list to realistically receive an organ in time. However, this procedure is commonly used in Japan with excellent outcomes reported.

\section{Immunosuppression}

Immunosuppressive regimens vary between transplant centers. About half of the lung transplant centers throughout the world use induction in the hopes of preventing early acute rejection. There is also a great deal of variability in the induction regimen of choice. Some centers will utilize an interleukin (IL)-2 receptor antagonist such as basiliximab. Other centers will use a lympholytic agent such as rabbit antithymocyte globulin (RATG) or equine antithymocyte globulin (ATGAM). These preparations are derived from animal serum and contain antibodies to human lymphocytes that can induce opsonization and phagocytosis of $\mathrm{T}$ lymphocytes, modulating their activation. 
Table 74.4 Immunosupressive agents used in pediatric lung transplantation

\begin{tabular}{|c|c|}
\hline Class of drug & Potential side effects \\
\hline \multicolumn{2}{|c|}{ Calcineurin inhibitors } \\
\hline Tacrolimus & $\begin{array}{l}\text { Infection, hyperglycemia, } \\
\text { hypertension, seizure, renal } \\
\text { dysfunction, PTLD }\end{array}$ \\
\hline Cyclosporine & $\begin{array}{l}\text { infection, hirsutism, gingival } \\
\text { hyperplasia, hypertension, seizures, } \\
\text { renal dysfunction, PTLD }\end{array}$ \\
\hline \multicolumn{2}{|c|}{ Cell cycle toxin inhibitors } \\
\hline $\begin{array}{l}\text { Mycophenolate } \\
\text { mofetil }\end{array}$ & $\begin{array}{l}\text { Infection, leukopenia, vomiting, } \\
\text { diarrhea, hepatic dysfunction, PTLD }\end{array}$ \\
\hline Azathioprine & Infection, leukopenia, PTLD \\
\hline \multicolumn{2}{|l|}{ mTOR inhibitors } \\
\hline Sirolimus & $\begin{array}{l}\text { Infection, delayed wound healing, } \\
\text { hypertriglyceridemia, interstial lung } \\
\text { disease, PTLD }\end{array}$ \\
\hline Everolimus & $\begin{array}{l}\text { Infection, interstitial lung disease, } \\
\text { renal dysfunction, stomatitis, } \\
\text { leukopenia }\end{array}$ \\
\hline
\end{tabular}

Because of the relative high frequency of acute rejection among lung transplant recipients, a maintenance strategy of triple drug immunosuppression is commonly employed (Table 74.4). The three drugs will typically consist of a calcineurin inhibitor (CNI), a cell cycle toxin inhibitor, and systemic corticosteroids. In pediatrics especially, we attempt to minimize steroid exposure, and during the first post-transplant year we wean the steroid dose from $0.5 \mathrm{mg} / \mathrm{kg}$ to $0.2 \mathrm{mg} /$ $\mathrm{kg}$; at our center it typically remains at that dose.

\section{Post-operative Monitoring and Management}

After the procedure is completed, patients are transferred to the intensive care unit, still intubated and on mechanical ventilator support, with some patients still requiring ECMO. Monitoring must require at minimum cardiorespiratory monitoring, including pulse oximetry, careful measurement of fluid input and output, and measurement of arterial blood gases. While initial management of ventilator support and ECMO, if needed, is done by the intensivist, all aspects of care are best delivered through a team approach, where the intensivist, the cardiothoracic surgeon, and transplant pulmonologist discuss all important aspects and meet at least once if not twice daily to round as a group. Some key aspects of post-operative care are discussed below, as well as in the subsequent section on complications that occur both early and late after lung transplantation. More in-depth discussion of all aspects can be found in the references.

\section{Fluids and Nutrition Management}

Owing to both capillary leak and interrupted lymphatic drainage, the transplanted lungs are particularly fluid sensitive, and thus reaching a negative fluid balance within the first 48 hours post-operatively is important. Colloids and pressors should be used to reduce the amount of stress placed on the kidneys, as overly aggressive diuresis, when combined with CNI nephrotoxicity, can quickly lead to renal failure, resulting in fluid overload and subsequent pulmonary compromise. Thus, while one will always use diuretics in this setting, one must do so carefully. Enteral nutrition should be started as soon as is appropriate but may be delayed for anticipated extubation or other procedures.

\section{Ventilator Management}

Foremost, the goal is to wean off the ventilator rapidly with the goal of extubation. The former should be done per the general practice of the experienced intensivist. Pressure-controlled ventilation is recommended because it places limits on peak airway pressures that protect not only the parenchyma but also the healing airway anastomoses. At our center, a bronchoscopy is performed 1 day post-operatively to inspect the anastomoses, and to obtain BAL if infection is suspected. Airway clearance is important in the initial post-operative period, with appropriate limits in place to avoid anastomotic complications. Physiotherapy and mobilization are also important, as these will not only help mobilize secretions but will also enhance chest tube drainage and alveolar recruitment. 


\section{Surgical Complications}

Airway anastomotic complications occur in $\sim 13 \%$ of recipients. They primarily manifest as stenosis with resulting airflow obstruction. This can generally be managed with bronchoscopic interventions. As the bronchi of children grow with the recipient, stents are avoided if possible in favor of repeated balloon dilation. Partial or complete dehiscence of the anastomosis is rarely seen but can be catastrophic.

Vascular anastomotic complications are also rare. At our center, a perfusion lung scan is routinely performed within 24 hours of the transplantation to evaluate the vascular anastomoses. Any significant discrepancy between right- and left-sided perfusion should be immediately evaluated in consultation with the cardiothoracic surgeons.

\section{Primary Graft Dysfunction}

Primary Graft Dysfunction (PGD) occurs within 72 hours of transplant and is characterized by non-specific alveolar injury, noncardiogenic pulmonary edema, and resulting decreased compliance and hypoxemia. It is caused by multiple factors, including ischemia-reperfusion injury, inflammation secondary to donor death, and other factors. In 2005, the ISHLT developed a classification system using chest $\mathrm{X}$-ray infiltrates and $\mathrm{PaO}_{2} / \mathrm{FiO}_{2}$ ratio to grade $\mathrm{PGD} 0-3$. On the basis of this classification, severe PGD $\left(\mathrm{PaO}_{2} / \mathrm{FiO}_{2}<200\right.$ at 48 hours post-transplant) occurs in $15 \%$ of recipients, which is concerning as severe PGD is associated with increased graft failure and mortality. Management of severe PGD is similar to the management of patients with acute respiratory distress syndrome (ARDS), including fluid restriction while maintaining perfusion to end organs, lung protective ventilator strategies, and nitric oxide. When the amount of oxygen and ventilator support is high and thus contributing to further lung injury, early ECMO is an important option to consider to both provide lung rest and minimize iatrogenic trauma to the graft.

\section{Post-transplantation Monitoring}

After the perioperative recovery period, recipients continue to require monitoring. Regular evaluation, initially at the transplant center, followed by close monitoring by a local physician with discussion with the transplant team, is necessary to identify and manage post-transplant complications. Routine monitoring includes measurement of CNI and antimetabolite levels, routine laboratory tests, pulmonary function testing, and chest imaging. While controversial, we believe that bronchoscopy with BAL and transbronchial biopsies by trained bronchoscopists an important part of post-transplant surveillance. Although scheduled surveillance bronchoscopy can detect asymptomatic acute rejection, allowing therapeutic interventions to occur sooner, a clear benefit in terms of outcomes has not been found. In infant lung transplant recipients, the use of surveillance bronchoscopy is more important as ambulatory lung function testing is not possible and patients are less able to report symptoms.

\section{Post-transplantation Complications}

\section{Infections}

Given the degree of immunosuppression needed to prevent acute rejection of the lung, infections are a predictable complication of lung transplantation. Selected infections are discussed in Table 74.5. However, virtually any pathogen, known and unknown, can present post-lung transplant. Clinicians must be vigilant and aggressive in managing these infections to preserve both graft function and the patient's well-being. A close working relationship with infectious disease specialists is extremely advantageous to lung transplant programs, with some programs including one on their transplant team. 
Table 74.5 Infections

Pathogen/infection

Bacterial

Bloodstream infections

Lower respiratory tract infections

Gram-negative bacteria (including multi-drug resistant isolates)

Burkholderia cenocepacia (genomvar III)

Burkholderia gladioli

Burkholderia multivorans

(Genomvar II)

Clostridium difficile

Viral

CMV

HSV

\section{VZV}

Respiratory viruses (including rhinovirus, influenza, parainfluenza, respiratory syncytial virus, and all other respiratory viruses)

Mycobacteria

Tuberculosis

Non-tuberculous mycobacteria

Fungi

Aspergillus \& candida

Pneumocystis jirovecii

Other fungi

\section{Notes}

Occur in $25 \%$ of recipients, usually early, when central lines are still in place. Occur across the course of time post-transplant, seen in $80 \%$ of recipients.

Cystic fibrosis recipients often have return of colonization from sinuses including resistant bacteria (P. aeruginosa, MRSA, etc.)

Absolute contraindication at nearly all centers due to poor post-transplant outcomes

Associated with increased post-transplant mortality, relative contraindication No contraindication, no increased risk

Occurs in 5-8\% of recipients, most frequently within the first 6 months posttransplant, re-appearing as a late complication ( $>2$ years post-transplant). Associated with significantly increased mortality, particularly when early post-transplant.

The most important viral infection post-transplant, can promote rejection. Requires months of antiviral prophylaxis (length is center specific) if either donor or recipient with positive serology, started at time of transplant. All recipients monitored using blood PCR, frequency depends on pre-transplant status.

Can be reactive post-transplant, thus if not on antiviral prophylaxis for CMV, and recipient or donor with history of HSV, consider prophylaxis with acyclovir or valacyclovir.

Immunization pre-transplant strongly recommended. If exposed, treat with varicella zoster immune globulin or intravenous immunoglobulin immediately.

Occur in more than half of recipients, often detected in the lower respiratory tract via bronchoscopy. Important to detect quickly and treat, as data suggest RSV can trigger rejection, data less clear for other viruses. Recommend recipients $<2$ years of age receive pavilizumab prophylaxis for RSV, all should get influenza vaccine yearly.

Increased risk of active infection post-transplant, $\sim 4 \%$ donor derived, others a combination of reactivation of latent TB and new infection. Tuberculin skin test and if $\geq 5$ years of age, Interferon Gamma Release Assay (i.e., Quantiferon Gold) recommended pre-transplant. Active infection should prompt discussion of delaying transplant until infection cleared. Latent infection requires 9 months isoniazid, pre-transplant if possible. Donor screening recommended. Treatment post-transplant challenging due to drug-drug interactions and diagnostic difficulty due to immunosuppression causing anergy

Mycobacterium avium-intracellulare is treatable; however, M. abscessus is a contraindication due to observed disseminated infection post-transplant, unless sputum can be cleared pre-transplant, chelonae is also treated this way.

Invasive fungal infections with Aspergillus and Candida can cause anastomotic complications, particularly early post-transplant, along with invasive pulmonary or disseminated disease. Detection requires vigilance; treatment requires aggressive approaches and consultation with infectious disease specialists. Antifungal azoles interact with calcineurin inhibitors and mTOR inhibitors, thus caution and close monitoring of drug levels must be used.

Post-transplant prophylaxis with TMP/SMX started no later than 3 weeks posttransplant for the life of the recipient. Alternative agents include dapsone and pentamidine. TMP/SMX also provides prophylaxis for Nocardia spp., and Toxoplasma gondii.

Cryptococcus and other fungi have been seen post-transplant and are important considerations in any illness of an immunosuppressed recipient 


\section{Post-transplant Lymphoproliferative Disease (PTLD)}

By 5 years post-transplant, $14 \%$ of pediatric recipients have had a malignancy of some type, nearly all of which are PTLD. The most common form of PTLD is caused by Epstein-Barr virus infection causing transformation of a CD20 cell, resulting in a B-cell lymphoma, most commonly polymorphic. Patients who develop primary EBV infection post-transplant are at greatest risk. For CD20 positive PTLD, anti-CD20 antibody (rituximab) therapy has revolutionized the management and allows successful treatment when combined with regimens of low-dose chemotherapy and reduced baseline immunosuppression. This approach causes far less toxicity than prior treatment options. Prognosis is substantially worse for CD20-negative PTLD, due to a combination of a lack of good therapeutic options and the toxicity of the available regimens. Overall, per the ISHLT registry (1/1992-6/2012), PTLD caused $3.3 \%$ of deaths in pediatric lung recipients, with other malignancies causing $0.8 \%$ of deaths.

The major challenge in PTLD is diagnosis, as it can present with anything from asymptomatic pulmonary nodules on CT scan, neurological or gastrointestinal symptoms, bone pain, or sinus pain. Further, although peak incidence is at 6 months post-transplant, it can be seen as a late complication as well. Thus, careful evaluation and assessment, including biopsies and imaging, are needed to make the diagnosis.

\section{Gastrointestinal Complications}

Gastrointestinal complications are seen in up to $50 \%$ of recipients. Clostriduim difficile is an important consideration, covered previously. Other GI issues seen include esophagitis, pancreatitis, gastroparesis, ileus, CMV infections of the gut and liver, cholecystitis, peptic ulcer disease, gastroesophageal reflux, and peptic ulcer disease. Patients with cystic fibrosis continue to be at risk for distal intestinal obstruction syndrome and must continue to take pancreatic enzyme supplements, along with bowel regimens (i.e., lactulose or Miralax), particularly in the early post-operative period to avoid obstruction. Gastroesophageal reflux deserves special mention, as data suggest that diagnosis and treatment of GER with antireflux surgery may prevent the development of bronchiolitis obliterans and ameliorate its progression if present, but these data have not been replicated in children. As mentioned above, PTLD can present as intussusception, obstruction, bowel perforation, feeding intolerance or abdominal pain. For GI complications, medical management for resolution is preferred. If this is not possible, elective surgical procedures are well tolerated, while data suggests that emergent surgical procedures carry a high morbidity and mortality risk.

\section{Neurological Complications}

Forty-seven percent of recipients have some neurological complications. The most common complication is seizures, generally felt to be related to calcineurin inhibitor induced cerebral vasoconstriction. Posterior reversible encephalopathy syndrome (PRES) can also be seen. PRES consists of seizure activity in $92 \%$ of cases, along with headaches, visual abnormalities, nausea/vomiting, other focal neurological signs, and varying degrees of impaired consciousness, ranging from confusion, somnolence, and lethargy to encephalopathy or coma. The diagnosis requires both symptoms and neuroimaging findings, with MRI being superior to CT scan for identifying PRES. Consultation with a neurologist is also recommended; however, long-term medication is not generally needed for seizures related to lung transplantation. Hypertension can be present and contributory, and should be managed accordingly. While calcineurin inhibitor toxicity is felt to contribute to both seizures and PRES, the correlation with levels and severity or incidence is incomplete. Although some patients respond to switching CNI (i.e., from tacrolimus to cyclosporine or vice versa) or alternative regimens, no general recommendation exists. 


\section{Diabetes}

Diabetes is seen in $20 \%$ of recipients in 1 year, $30 \%$ at 5 years, and is seen most commonly in patients with cystic fibrosis and is thus likely related to pre-existing and ongoing pancreatic islet cell injury. Tacrolimus and corticosteroids both appear to increase the risk of diabetes.

\section{Renal Dysfunction}

Nearly all recipients develop hypomagnesemia post-transplant, with some developing renal tubular acidosis, and both are generally managed with oral supplements. Early renal failure suggests pre-transplant renal insufficiency. Thus, measurement of glomerular filtration rate is a mandatory aspect of pre-transplant evaluation, as patients with GFR $<50$ have a suspected risk for poor outcomes, and it thus represents a contraindication to transplant at some centers. Chronic effects of exposure to CNI and resulting renal vasoconsctriction contributes significantly to renal insufficiency and renovascular hypertension post-transplant. Generally, this is managed with calcium channel blockers. Five years of CNI based immunosuppression carries a $2.3 \%$ incidence of renal failure requiring dialysis, and $1 \%$ require renal transplantation at some point post-transplant.

\section{Rejection}

\section{Acute Rejection}

Acute cellular rejection is common in the first weeks to months post-transplant. While often asymptomatic, fever, hypoxemia, and dyspnea can be seen. CXR findings include infiltrates and bilateral pleural effusions. Similarly, a 10\% decrease in either FEV1 or FVC on home spirometric monitoring should prompt further evaluation. Biopsies are graded per the 2007 Revision of the 1996 working formulation for the standardization of nomenclature in the diagnosis of lung rejection. The score consists of two components.
The A score reflects the intensity and composition of infiltrating immune cells and the extent of parenchymal involvement (A0, no rejection, A1-4 minimal to severe acute rejection). The B score reflects airway involvement.

Only acute rejection episodes graded A2 or higher are treated, generally with 3-4 days of pulse IV methylprednisolone (10-20 mg/kg/day), with follow-up biopsy in 2-4 weeks. Persistent acute rejection is treated with additional pulse steroids, anti-T-cell antibody therapy, and augmented baseline immunosuppression. Acute rejection is infrequent after the first year posttransplant and is less common in infants than in older children.

\section{Antibody Mediated Rejection}

While hyperacute antibody-mediated rejection caused by pre-formed anti-donor antibodies present at the time of transplant is a well established cause of acute graft failure, it is rare. Antibody mediated rejection (AMR) outside that context is now recognized as an important complication in lung transplantation. As diagnostic criteria are still evolving, and retrospective evaluations in progress, the exact incidence of AMR in pediatric lung transplantation is as yet unknown. AMR can present as progressive worsening of lung function in the absence of acute or chronic rejection, and can rapidly result in death due to graft failure.

Currently, diagnosis of AMR requires three features be present, including evidence of allograft dysfunction, presence of circulating donor-specific antibodies, and histopathologic findings of AMR. However, this is complicated by the significant overlap of these patterns with pathology seen in other forms of lung injury, including high-grade or persistent acute cellular rejection, diffuse alveolar damage, high-grade or persistent lymphocytic bronchiolitis, obliterative bronchiolitis, arteritis without rejection, or other graft dysfunction without clear morphological explanation.

Treatment of antibody mediated rejection is still evolving. As such, the components of therapy, rather than specific regimens, will be dis- 
cussed. Plasmapheresis or therapeutic plasma exchange is a process for removal of antibodies and other circulating proteins from the recipient's bloodstream by filtration and thus directly removes the responsible antibodies. Pulse corticosteroids are also generally used and have been found to impair T-cell and B-cell function, impair antibody and complement binding, and decrease levels of serum immunoglobulins. Rituximab, an anti-CD20 antibody used for PTLD, is also used in AMR, as it targets the B-cells that make antibody. Its use in AMR mirrors its successful use in antibody-mediated autoimmune diseases such as rheumatoid arthritis. Together, these three interventions make up the initial treatment of AMR.

In summary, while antibody mediated rejection is clearly an important process, the exact pathobiology, incidence, diagnostic approach, treatment, and understanding outcomes remain in evolution. Ongoing studies will help to determine how to best diagnose and treat this entity in pediatric lung recipients.

\section{Chronic Lung Allograft Dysfunction}

Chronic lung allograft dysfunction (CLAD) is a newer term used to define the varying patterns of long-term respiratory decline and failure seen in lung recipients. It encompasses both bronchiolitis obliterans and restrictive long-term allograft dysfunction.

Acute lung allograft dysfunction (ALAD) is defined as any acute cause of a drop in lung function, defined as a $10 \%$ drop from baseline in post-bronchodilator FEV1 and/or FVC, including acute rejection and infection. If this persists for more than 3 weeks, it is termed suspected CLAD, and evaluation should include bronchoscopy with biopsies. Should these prove nondiagnostic, and should lung function not recover, open lung biopsy is also an important consideration in pediatric recipients, particularly smaller children in which the diagnostic yield of transbronchial biopsies is limited by the use of small forceps. If there is evidence of persistent acute rejection, infection, anastomotic complication, diaphragm dysfunction, or other causes, CLAD is not diagnosed.

CLAD is divided into restrictive CLAD and obstructive CLAD. Restrictive CLAD, also called restrictive allograft syndrome, is named for the pulmonary function picture, which includes TLC $\leq 90 \%$ baseline and/or FEV1/FVC normal or increased, with FEV1 and/or FVC decline $\leq$ $80 \%$ of baseline. HRCT findings will include infiltrates, ground glass opacities, and upper lung zone fibrotic changes. Restrictive CLAD (R-CLAD) carries a significantly worse prognosis than BOS, with median survival of 541 days for patients with R-CLAD compared to 1421 for patients with BOS.

After 3 years post-transplant, obstructive CLAD or BOS becomes the major cause of death in pediatric recipients, and at 5 years, $50 \%$ of surviving recipients carry a diagnosis of BOS. In obstructive CLAD or BOS, the picture is dominated by the drop in FEV1, and FVC and TLC are stable or significantly less affected. HRCT findings will include air trapping.

In terms of diagnosing BOS, the new CLAD classification system overlaps with the prior definitions of bronchiolitis obliterans syndrome (BOS). For example, BOS $0 p$ is defined as a $\geq$ 10 but $<20 \%$ drop in post-bronchodilator FEV1 from baseline or FEF $25-75<75 \%$ of baseline. In this group there appears to be a group of patients with what has been termed azithromycinresponsive allograft dysfunction (ARAD). Azithromycin entered use in bronchiolitis obliterans syndrome in the early 2000s, after it was noted that patients on thrice-weekly azithromycin, as used in cystic fibrosis, could have stabilization and even improvement of lung function in the setting of bronchiolitis obliterans, despite lack of a clear mechanism of action, though both anti-inflammatory and anti-fibrotic activities of azithromycin are posited as being responsible for this therapeutic efficacy.

Once pulmonary function testing suggests BOS, either by a drop in FEV1, or a drop in FEF25-75, which has been shown to be more sensitive and detects small airways dysfunction earlier, diagnosis requires biopsy to confirm the presence of small airway obliteration by a fibrotic 
process. Currently, open or thoracoscopic lung biopsy is used at our center when BOS is suspected but cannot be confirmed by transbronchial biopsies.

Treatment of BOS is varied, and over time has included use of augmented immunosuppression, change in baseline immunosuppression, azithromycin, extracorporeal photopheresis (ECP), and total lymphoid irradiation. Currently, our practice is to use pulse corticosteroids accompanied by 10 days of anti-thymocyte antibodies, along with initiation of azithromycin thrice-weekly. If this does not stabilize symptoms, ECP is initiated. This is consistent with the current best evidence. While inhaled cyclosporine and mTOR inhibitors have both been shown to be effective in small studies, larger trials have not found a consistent benefit in the use of either to prevent the development of BOS, or in its treatment. Surgical treatment of gastroesophageal reflux has been shown to be beneficial in adults in managing and preventing BOS, but this has not been replicated in children. Similarly, retrospective reviews suggest that statin therapy can prevent BOS and may be beneficial in treating BOS.

\section{Outcomes}

Outcomes in lung transplantation as a whole continue to lag behind those of other solid organ transplants, and this is also true in children. While 1-year survival rates have improved to $\sim 85 \%, 3$ year survival is $\sim 65 \%$, and median survival is 4.9 years, lower but not statistically significantly different than the 5.4 year median survival in adult recipients. As discussed previously, long-term survival is determined primarily by the development of BOS. While retransplantation continues to be offered at a small number of pediatric centers for allograft failure, outcomes are worse for retransplantation than primary transplantation ( $45 \%$ versus $58 \%$ survival at 3 years post-transplant), irrespective of whether or not re-transplantation was for BOS or other causes of graft failure. Data suggest that patients re-transplanted at least 1 year after their primary transplant had better outcomes, but the numbers of re-transplants are too small to make a definitive conclusion. Further, these outcomes have been relatively stable over the past 10 years, demonstrating the urgent need for new ways of managing recipients in order to preserve graft function and ultimately life.

\section{Future Considerations}

Several areas of active research offer the possibility of improved outcomes in lung transplantation. One such area is the expansion of donor lung availability using ex vivo lung perfusion. In this procedure, marginal donor lungs that are not accepted for transplant initially are ventilated and perfused ex vivo and allowed to recover. Lungs have been successfully transplanted this way, and expanding the donor pool provides the opportunity to save patients that still, at present, die waiting for organs. This procedure needs to be extended into children as soon as possible. Another such area is the use of pumpless oxygenators as a bridge to lung transplantation. In conventional ECMO, a pump takes blood from the patient, into the oxygenator then returns it. Recently, pumpless oxygenator systems have been used in candidates awaiting lung transplantation (i.e., NovaLung). While pediatric specific systems are still being developed, we have used a neonatal membrane oxygenator (Quadrox) to successfully bridge an infant suffering from alveolar capillary dysplasia to transplant. Both pumpless oxygenators and venovenous ECMO are being increasingly used as ways to less invasively bridge candidates to transplant for longer periods of time than conventional ECMO, which requires significant sedation, mechanical ventilation, and immobility, which while buying some time, also leads to progression of the primary lung disease, all of which limit the time a candidate can be bridged on conventional ECMO. Last, an array of anti-cytokine therapeutic antibodies being developed for other therapeutic indications (i.e., anti-IL-13, anti-IL-5R, anti-IL-17, and others) provide an opportunity to manipulate these mediators, all seen in different forms and stages of lung transplant rejection, in order to treat or 
prevent rejection, and potentially to promote engraftment.

In pediatrics, the small number of recipients worldwide limits performing adequately powered trials. While this is not the case in adult recipients, trials are generally done at single centers, and this has resulted in underpowered studies that have failed to show differences between therapies that have beneficial trends (i.e., everolimus compared to MMF, inhaled cyclosporine). Multicenter trials should become the standard in lung transplantation as they have in many other indications. In pediatrics, the International Pediatric Lung Transplant Collaborative works to make this a reality. As outcomes from BOS remain poor despite therapies, with BOS causing $40 \%$ of all mortality beyond the first year of transplant, such studies, along with work in recently established animal models of orthotopic lung transplantation, are desperately needed to improve outcomes in pediatric lung transplantation.

\section{Sources}

Belperio JA, Weigt SS, Fishbein MC, Lynch JP 3rd. Chronic lung allograft rejection: mechanisms and therapy. Proc Am Thorac Soc. 2009;6:108-21.

Benden C, Edwards LB, Kucheryavaya AY, Christie JD, Dipchand AI, Dobbels F, Kirk R, Lund LH, Rahmel AO, Yusen RD, Stehlik J. The Registry of the International Society for Heart and Lung Transplantation: sixteenth official pediatric lung and heart-lung transplantation report-2013; focus theme: age. International Society for Heart and Lung Transplantation. J Heart Lung Transplant. 2013;32(10):989-97.

Choong CK, Sweet SC, Guthrie TJ, Mendeloff EN, Haddad FJ, Schuler P, De La Morena M, Huddleston $\mathrm{CB}$. Repair of congenital heart lesions combined with lung transplantation for the treatment of severe pulmonary hypertension: a 13-year experience. J Thorac Cardiovasc Surg. 2005;129(3):661-9.

Christie JD, Carby M, Bag R, Corris P, Hertz M, Weill D, ISHLT Working Group on Primary Lung Graft Dysfunction. Report of the ISHLT Working Group on Primary Lung Graft Dysfunction part II: definition. A consensus statement of the International Society for Heart and Lung Transplantation. J Heart Lung Transplant. 2005a;24:1454-9.

Christie JD, Van Raemdonck D, de Perrot M, Barr M, Keshavjee S, Arcasoy S, Orens J. ISHLT Working Group On Primary Lung Graft Dysfunction. Report of the ISHLT Working Group On Primary Lung Graft
Dysfunction part I: introduction and methods. J Heart Lung Transplant. 2005b;24:1451-3.

Elizur A, Faro A, Huddleston CB, Gandhi SK, White D, Kuklinski CA, Sweet SC. Lung transplantation in infants and toddlers from 1990 to 2004 at St. Louis Children's Hospital. Am J Transplant. 2009;9(4):719-26.

Estenne M, Maurer JR, Boehler A, Egan JJ, Frost A, Hertz M, Mallory GB, Snell GI, Yousem S. Bronchiolitis obliterans syndrome 2001: an update of the diagnostic criteria. J Heart Lung Transplant. 2002;21:297-310.

Faro A, Hamvas A. Lung transplantation for inherited disorders of surfactant metabolism. NeoReviews. 2008;9:e468-76.

Faro A, Mallory GB, Visner GA, Elidemir O, Mogayzel PJ Jr, Danziger-Isakov L, Michaels M, Sweet S, Michelson P, Paranjape S, Conrad C, Waltz DA. American Society of Transplantation executive summary on pediatric lung transplantation. Am J Transplant. 2007;7(2):285-92.

Grady RM, Gandhi S, Sweet SC, Mao J, Huddleston CB. Dismal lung transplant outcomes in children with tetralogy of Fallot with pulmonary atresia compared to Eisenmenger syndrome or pulmonary vein stenosis. J Heart Lung Transplant. 2009;28(11):1221-5.

Gupta S, Fricker FJ, González-Peralta RP, Slayton WB, Schuler PM, Dharnidharka VR. Post-transplant lymphoproliferative disorder in children: recent outcomes and response to dual rituximab/low-dose chemotherapy combination. Pediatr Transplant. 2010;14:896-902.

Huddleston CB. Donor evaluation, surgical technique and perioperative management. In: Fine RN, Webber SA, Olthoff KM, Kelly DA, Harmon WE, editors. Pediatric solid organ transplantation. 2nd ed. Malden: Blackwell Publishing; 2007. p. 326-35.

Kerem E, Reisman J, Corey M, Canny GJ, Levison H. Prediction of mortality in patients with cystic fibrosis. N Engl J Med. 1992;326(18):1187-91.

Lim GY, Newman B, Kurland G, Webber SA. Posttransplantation lymphoproliferative disorder: manifestations in pediatric thoracic organ recipients. Radiology. 2002;222:699-708.

Liou TG, Adler FR, Cox DR, Cahill BC. Lung transplantation and survival in children with cystic fibrosis. $\mathrm{N}$ Engl J Med. 2007;357(21):2143-52.

Mendeloff E. Historical notes. In: Fine RN, Webber SA, Olthoff KM, Kelly DA, Harmon WE, editors. Pediatric solid organ transplantation. 2nd ed. Malden: Blackwell Publishing; 2007. p. 309-13.

Rivera-Spoljaric K, Faro A. Fibrosis quística: cuando referir para trasplante pulmonar pediatrico. Neumol Pediatr. 2007;2(2):84-9.

Schuurmans MM, Benden C, Inci I. Practical approach to early postoperative management of lung transplant recipients. Swiss Med Wkly. 2013;143:w13773.

Sharples LD, McNeil K, Stewart S, Wallwork J. Risk factors for bronchiolitis obliterans: a systematic review of recent publications. J Heart Lung Transplant. 2002;21:271-81. 
Stegall MD, Gloor JM. Deciphering antibody-mediated rejection: new insights into mechanisms and treatment. Curr Opin Organ Transplant. 2010;15:8-10.

Stewart S, Fishbein MC, Snell GI, et al. Revision of the 1996 working formulation for the standardization of nomenclature in the diagnosis of lung rejection. $\mathrm{J}$ Heart Lung Transplant. 2007;26:1229-42.

Sweet SC, Aurora P, Benden C, Wong JY, Goldfarb SB, Elidemir O, Woo MS, Mallory GB, International
Pediatric Lung Transplant Collaborative. Lung transplantation and survival in children with cystic fibrosis: solid statistics-flawed interpretation. Pediatr Transplant. 2008;12(2):129-36.

Yusen RD, Shearon TH, Qian Y, Kotloff R, Barr ML, Sweet S, Dyke DB, Murray S. Lung transplantation in the United States, 1999-2008. Am J Transplant. 2010;10(4:Pt 2):t-68. 